\title{
Discours
}

Revue de linguistique, psycholinguistique et

informatique. A journal of linguistics, psycholinguistics and computational linguistics

$22 \mid 2018$

Varia

\section{Topique et focus discursifs locaux chez les enfants et les adultes hongrois}

\section{Gabriella Fekete}

\section{OpenEdition}

Journals

Édition électronique

URL : http://journals.openedition.org/discours/9611

DOI : 10.4000/discours.9611

ISSN : 1963-1723

Éditeur :

Laboratoire LATTICE, Presses universitaires de Caen

\section{Référence électronique}

Gabriella Fekete, "Topique et focus discursifs locaux chez les enfants et les adultes hongrois », Discours [En ligne], 22 | 2018, mis en ligne le 19 octobre 2018, consulté le 01 mai 2019. URL : http:// journals.openedition.org/discours/9611 ; DOI : 10.4000/discours.9611 

Revue de linguistique, psycholinguistique et informatique

\title{
Topique et focus discursifs locaux chez les enfants et les adultes hongrois
}

\author{
Gabriella Fekete \\ Dynamique du langage (DDL) \\ CNRS \\ Université de Lyon
}

Gabriella Fekete, «Topique et focus discursifs locaux chez les enfants et les adultes hongrois», Discours [En ligne], 22 | 2018, mis en ligne le 19 octobre 2018.

URL: http://journals.openedition.org/discours/9611

Titre du numéro: Varia

Coordination: Benjamin Fagard \& Denis Vigier

Date de réception de l'article: 25/02/2018

Date d'acceptation de l'article: 05/06/2018 



\title{
Topique et focus discursifs locaux chez les enfants et les adultes hongrois
}

\author{
Gabriella Fekete \\ Dynamique du langage (DDL) \\ CNRS \\ Université de Lyon
}

Dans cette étude, nous nous intéressons à la trajectoire développementale du topique et du focus discursifs locaux en hongrois. Nous défendons l'hypothèse qu'en hongrois, le focus discursif local véhicule l'information placée au centre d'attention (Rickheit et Habel, 1995) et se trouve toujours en position initiale de la proposition. Le topique discursif local relie l'information au contexte (Cornish, 2004) et n'est pas toujours placé en position initiale. Ce phénomène aboutit à des associations (topique focalisé en position initiale) et à des dissociations topique-focus (le focus et le topique sont deux arguments différents) dans les textes hongrois. Afin d'observer la trajectoire développementale de celles-ci, nous avons collecté des narrations orales auprès de locuteurs hongrois natifs appartenant à 5 groupes d'âge $(3,5$, 7-8, 11-12 ans, adultes). Le stimulus utilisé était le livre Frog, where are you? (Mayer, 1969). Nos résultats confirment le consensus général sur la préférence des locuteurs pour les topiques focalisés en position initiale dans les textes narratifs. Toutefois, nos observations montrent que le taux des dissociations augmente avec l'âge. Les topiques dissocié et associé sont utilisés pour le maintien des personnages récurrents à l'aide des suffixes verbaux. Les enfants attirent l'attention surtout sur les actions jusqu'à l'âge de 7-8 ans alors que les adolescents et les adultes recourent au rôle de focus dissocié pour introduire des entités inanimées et pour réintroduire les personnages primaires.

Mots clés: topique discursif, focus discursif, étude développementale, narration orale, hongrois

In the present study, we would like to observe the development of local discourse topic and focus in Hungarian. We propose that, in Hungarian, the local discourse focus not only conveys information placed at the center of attention (Rickheit \& Habel, 1995), but is also always positioned at the beginning of the clause. The local discourse topic determines the point of view and links information to context (Cornish, 2004), but it has no dedicated position in the sentence. This phenomenon leads to associations (foregrounded topic in clause-initial position) and dissociations (focus and topic are two different arguments) of topic-focus status in Hungarian texts. In order to observe the developmental trajectory of topic and focus, we collected oral narrations from native Hungarian speakers of 5 age groups (3, 5, 7-8, 11-12 year-olds and adults). The stimulus used was the book Frog, where are you? (Mayer, 1969). Our results show that in narrative texts, Hungarian speakers prefer to put the topic-focus association in the initial position. By contrast, the rate of dissociation increases with age. Dissociated and associated topics are used to maintain recurrent characters with verbal suffixes. For children up to 7-8 years old, the use of dissociated focus mainly includes actions, while adolescents and adults use this role both to introduce inanimate entities and to reintroduce primary characters.

Keywords: discourse topic, discourse focus, developmental study, oral narratives, Hungarian 


\section{Abréviations}

3

ABL

ACC

ADJ

ADV

COND

D

DAT

DEF

[Déict]

DEL

ELA

[F]

[F+T]

FREQ

G

ILL

INDEF troisième personne

ablatif

accusatif

suffixe adjectival

suffixe adverbial

conditionnel

conjugaison définie

datif

défini

déictique

délatif

élatif

élément en focus

élément en focus et en topique

fréquentatif

conjugaison générale

illatif

indéfini

$\begin{array}{ll}\text { INE } & \text { inessif } \\ \text { INF } & \text { infinitif } \\ \text { MOY } & \text { voix moyenne } \\ \text { NEG } & \text { négation } \\ \text { P } & \text { pluriel } \\ \text { PAS } & \text { passé } \\ \text { POS } & \text { possessif } \\ \text { POT } & \text { potentiel } \\ \text { PROP } & \text { pronom personnel } \\ \text { PRS } & \text { présent } \\ \text { PV } & \text { préverbe } \\ \text { REL } & \text { relatif } \\ \text { S } & \text { singulier } \\ \text { SUB } & \text { sublatif } \\ \text { SUP } & \text { superessif } \\ \text { [T] } & \text { élément en topique } \\ \text { TER } & \text { terminatif }\end{array}$

\section{Introduction}

La transmission verbale d'une information implique de faire un choix entre plusieurs options linguistiques (exemple [1]) pour l'organisation du flux de l'information (Jisa et al., 2002).

[1] a. Le garçon, le chien l'effraye.

b. Le chien est effrayant.

c. Le garçon est effrayé (par le chien).

d. Le chien effraye le garçon.

Nous choisissons la structure qui rend notre représentation mentale de l'information le plus fidèlement possible, en prenant en compte les connaissances de notre interlocuteur. Ainsi, nous mettons certains éléments au premier plan (le chien dans [1a-b], le garçon dans [1c-d]), ce qui en repousse d'autres à l'arrière-plan. À travers cette sélection complexe, nous amenons l'interlocuteur à la même interprétation subjective de l'information.

Nous pouvons gérer ce processus de mise en avant et en arrière-plan à l'aide de divers moyens linguistiques tels que la transitivité (exemple [2]), la forme référentielle 
(exemple [3]), la voix grammaticale (exemple [4]) ainsi que l'ordre des mots et l'organisation informationnelle topique-focus (exemple [5]) (Berman et Slobin, 1994).

[2] a. Le chien est effrayant.

b. Le chien effraye le garçon.

[3] a. Il l'effraye.

b. Le chien l'effraye.

c. Il effraye le garçon.

[4] Le garçon est effrayé.

[5] Le garçon, le chien l'effraye.

Le traitement de ces moyens implique une coordination entre le niveau de la phrase et celui du discours (Hickmann, 1995).

Dans la présente étude, nous nous intéressons à l'organisation informationnelle topique-focus avec un double objectif: nous souhaitons (i) clarifier les notions de topique et de focus afin (ii) d'observer la trajectoire développementale du topique et du focus discursifs locaux en hongrois. À notre connaissance, aucune étude développementale n'a encore pris en compte la relation phrase-discours et l'articulation topique-focus dans le discours en hongrois. Il est particulièrement intéressant d'aborder ce phénomène dans cette langue à sujet (pro-drop) et objet nuls et dont la structure phrastique est déterminée par l'articulation logico-sémantique «sujet logique-prédicat logique» (Gécseg et Kiefer, 2009).

Nous présenterons tout d'abord la problématique liée aux notions de topique et de focus (section 2). Ensuite, nous exposerons les caractéristiques principales de la langue hongroise (section 3.1) et nous formulerons les définitions adoptées dans cette étude (section 3.2). Après avoir donné un aperçu global sur les études développementales ayant traité de l'organisation informationnelle (section 4), nous exposerons nos hypothèses (section 5) et la méthodologie utilisée pour l'observation développementale (section 6). Enfin, nous présenterons les principaux résultats (section 7) pour terminer par une conclusion globale (section 8).

\section{Topique et focus : problématique terminologico-conceptuelle}

7 Selon la littérature, le topique représente l'entité, connue du contexte/de la situation (Kiss, 2002; Kiss et al., 2003), à propos de laquelle le reste de la phrase apporte une nouvelle information (Hajičová, 2007; Reinhart, 1981; Strawson, 1964). Cette entité est le sujet logique/psychologique du prédicat (Kiss, 2008; Gabelentz, 1869) et s'avère souvent être identique au sujet grammatical de la phrase et/ou à l'agent de l'action (Chafe, 1987; Givón, 1992; Lambrecht, 2000). Elle se trouve en position initiale de la phrase (Kiss, 2007; Rizzi, 1997). 
[6] [ Le garçon], le chien l'effraye.

La littérature mentionne deux types de focus: le focus informationnel ou présentationnel et le focus identifiant ou contrastif (Kiss, 1998; Rizzi, 1997; Zubizarreta, 1998). Nous nous intéressons ici au focus informationnel. Celui-ci fournit l'information principale et non présupposée sur le topique (Gundel et Fretheim, 2004) mais n'exprime pas d'identification exhaustive. Il véhicule un accent tonal. Il n'a ni restriction au niveau de la définitude, ni place spécifique dans la phrase (Kiss, 1998).

\section{[7] Le chien effraye [F le garçon].}

De nombreuses études (Galmiche, 1992; Gécseg et Kiefer, 2009; LikhachevaPhilippe, 2010; Mondada, 1994; Grobet, 2002) ont signalé la complexité conceptuelle de ces deux notions, liée à l'intrication de différents niveaux d'analyse: syntaxique, sémantique, discursif, cognitif et psychologique. Pour Galmiche (1992), au carrefour de cette confusion se trouve l'interaction entre facteurs linguistiques et discursifs intervenant dans l'organisation informationnelle. Il est donc important de distinguer: (i) la terminologie phrastique qui représente l'ensemble des procédés qui peuvent marquer l'articulation de la phrase, (ii) l'analyse discursive locale qui renferme les relations informationnelles phrase-contexte, et (iii) le traitement discursif global qui se déroule au niveau du sens global du discours (Creissels, 2006; Dik, 1997; Likhacheva-Philippe, 2010; López, 2009; Moya, 2006; Neeleman et Vermeulen, 2012; Tolcsvai Nagy, 2001; Van Dijk, 1977 et 1981; Van Dijk et Kintsch, 1983; Van Kuppevelt, 1995).

Dans la suite de l'étude, nous nous intéressons au topique et au focus discursifs locaux.

\section{Topique et focus discursifs locaux en hongrois}

\subsection{Les caractéristiques du hongrois}

11 Le hongrois est une langue au caractère agglutinant: de nombreux morphèmes marquent chacun une fonction grammaticale (exemple [8]). Ainsi, chaque argument est muni d'un suffixe casuel en fonction de sa relation grammaticale. Kiefer (1987) comptabilise 18 cas nominaux.

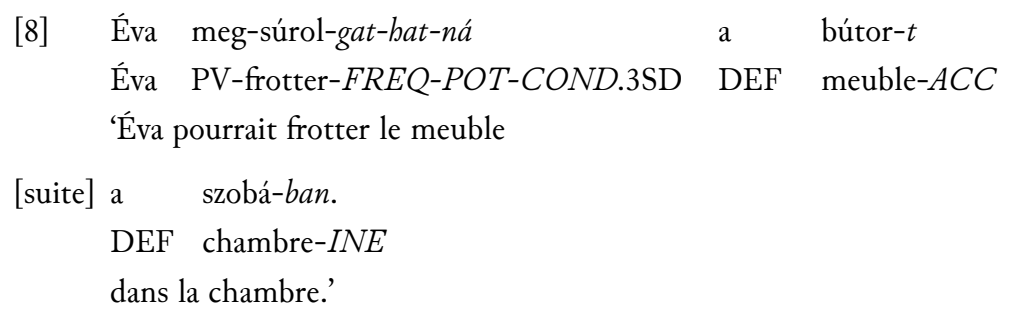


En outre, le hongrois est une langue à sujet nul, dite "pro-drop» car chaque verbe conjugué porte la marque du sujet et fournit des informations suffisantes sur celui-ci. De ce fait, l'utilisation des pronoms personnels est destinée uniquement à l'accentuation.

Le verbe transitif hongrois possède une double conjugaison qui peut être partiellement mise en corrélation avec la définitude du complément d'objet direct (Coppock et Wechsler, 2012; Den Dikken, 2006; Kiss, 2002 ; Kiefer et Kiss, 1994). La conjugaison appelée générale (ou subjective) (exemple [9a]) est utilisée avec un verbe intransitif ou un verbe transitif accompagné d'un objet indéfini. Le verbe transitif avec un objet défini requiert la conjugaison définie (ou objective) (exemple [9b]).

$\begin{array}{llllll}\text { [9a] A } & \text { lány } & \text { néz } & \text { egy } & \text { film-et. } \\ & \text { DEF } & \text { fille } & \text { regarder.PRS.3SG } & \text { INDEF } & \text { film-ACC }\end{array}$

'La fille regarde un film.'

$\begin{array}{llllll}\text { [9b] A } & \text { lány } & \text { néz-i } & a & \text { film-et. } \\ & \text { DEF } & \text { fille } & \text { regarder-PRS.3SD } & \text { DEF } & \text { film-ACC }\end{array}$

'La fille regarde le film.'

La structure de la phrase hongroise n'est pas déterminée par la fonction syntaxique des constituants mais par l'articulation logico-sémantique du sujet logique et du prédicat logique (Gécseg et Kiefer, 2009) (exemple [10]).

[10] a. János látja Marit. ('János voit Mari.')

b. Marit látja János.

c. János Marit látja.

d. Látja János Marit.

Ce sont ces propriétés morphologiques (sujet et objet nuls) et pragmatiques (ordre des constituants syntaxiques flexible) qui gouvernent l'organisation de l'information.

\subsection{Topique et focus dans le discours hongrois}

Nous soutenons l'idée généralement admise que le topique discursif identifie l'item linguistique à propos duquel une nouvelle information est donnée dans la proposition. Sa fonction consiste à déterminer la perspective de l'information et à fixer le point d'ancrage sémantique dans le discours (Cornish, 2004). Par conséquent, c'est un élément connu ou déductible du contexte. L'argument topical fait partie de l'arrière-plan de l'information, il est donc souvent omis ou pronominalisé puisqu'il est référentiel et identifiable à partir du contexte (exemple [11a]) (Gécseg et Kiefer, 2009; Lambrecht, 1994; Goldberg, 2004; Neeleman et al., 2009; Tolcsvai Nagy, 2001; Van Rooij et Schulz, 2017). Il est exprimé de façon explicite (par des nominaux) lorsqu'il y a un changement de topique (exemple [11b]). 
$\begin{array}{llllll}\text { [11a] } & {\left[\mathrm{F}_{\mathrm{F}+\mathrm{T}} \mathrm{Az}\right.} & \text { idő-s } & \text { néni }] & \text { nyugodt-an } & \text { köt-öget-ett, } \\ & \mathrm{DEF} & \hat{a} \text {-ADJ } & \text { dame-ACC } & \text { calme-ADV } & \text { tricoter-FREQ-PAS.3S }\end{array}$

'La vieille dame tricotait tranquillement,

[suite] amikor megijesztett- $\left.{ }_{\mathrm{F}+\mathrm{T}} \mathrm{e}\right]$ a macska.

lorsque PV-effrayer-PAS-3SD DEF chat

lorsque le chat l'a effrayée.'

[11b] [ ${ }_{\mathrm{F}+\mathrm{T}} \mathrm{Az}$ idő-s néni] nyugodt-an köt-öget-ett, DEF âge-ADJ dame-ACC calme-ADV tricoter-FREQ-PAS.3S

'La vieille dame tricotait tranquillement,

[suite] amikor ${ }_{\mathrm{F}+\mathrm{T}} \mathrm{a}$ macska] hirtelen le-ugr-ott

lorsque DEF chat soudain PV-sauter-PAS.3S

lorsque le chat a soudain sauté

[suite] a szekrény tete-jé-ről.

DEF armoire toit-POS-DEL

du haut de l'armoire.'

Le topique n’a pas de place dédiée (Gécseg et Kiefer, 2009; Givón, 2001 ; Lambrecht, 1994; Neeleman et al., 2009; Tolcsvai Nagy, 2006). Cependant, l'opération syntaxique qui consiste à déplacer un élément du groupe verbal vers une position syntaxiquement plus saillante (position initiale de la proposition) (Gécseg et Kiefer, 2009), pour le mettre au premier plan, s'appelle «topicalisation», «promotion» ou «mise en avant» (foregrounding). Un élément déplacé en position initiale devient-il topique grâce à ce déplacement? Pour notre part, nous soutenons que la place initiale ne topicalise pas l'élément mais le rend saillant au niveau pragmatique (exemple [12]) (Enkvist, 1989; Givón, 1992 ; Gernsbacher, 1995 ; Likhacheva-Philippe, 2010; Virtanen, 2004). Par conséquent, il ne s'agit pas de topicalisation mais de focalisation discursive.
[12a] Meg-ijeszt-ett- ${ }_{F+T}$ e] az idő-s néni-t. PV-effrayer-PAS-3SD DEF âge-ADJ dame-ACC
'Il [= le chat] a effrayé la vieille dame'.

[12b] $\left[{ }_{F} \mathrm{Az}\right.$ idős néni] meg-ijed-t [T tőle]. DEF âge-ADJ dame-ACC PV-prendre.peur.MOY-PAS-3S PROP3S-ABL 'La vieille dame a eu peur de lui [= du chat]'.

Dans l'exemple [12], le topique est le chat. Dans [12a], il est indexé sur le verbe et donc considéré comme focalisé alors que dans [12b], il n'est pas promu puisqu'il reste à sa place postverbale; c'est la «vieille dame» qui porte la saillance informationnelle.

De ce fait, nous proposons que le focus discursif renvoie à l'élément le plus saillant de l'information, qui se situe au centre d'attention immédiat du locuteur 
et de l'interlocuteur (Dik, 1997; Givón, 1988; Hajičová et Sgall, 1984; Hickmann, 1995; Likhacheva-Philippe, 2010; Tolcsvai Nagy, 2001; Stutterheim et Klein, 1989). Comme la place initiale a généralement un rôle primordial dans le flux de l'information, nous suggérons qu'elle véhicule le focus discursif en hongrois (Enkvist, 1989; Gernsbacher, 1995; Givón, 1992; Virtanen, 2004). Cet item linguistique peut transmettre une information nouvelle ou ancienne, il peut donc être plus ou moins accessible. La forme linguistique choisie pour l'expression du focus dépend de la présence de cet item dans le contexte discursif. Lorsque le focus comporte une information connue ou déductible du contexte précédent, les formes référentielles (zéro et pronominales) sont favorisées (exemple [11a]); lorsque nous avons affaire à une information nouvelle, ce sont les formes nominales qui servent à l'encodage linguistique du focus discursif (exemples [11b], [12]). Dans le premier cas, il s'agit de topique focalisé.

Cette approche nous amène à distinguer des associations et des dissociations du topique et du focus discursifs. Nous parlons d'association (exemples [11], [12a]) lorsque le topique est au centre d'attention immédiat du locuteur et de l'interlocuteur. Il est focalisé et déplacé en position initiale de la proposition. Dans les dissociations (exemple [12b]), le focus et le topique sont deux arguments différents: le focus se trouve à la place initiale et le topique à une place non initiale.

Nous défendons l'hypothèse qu'en hongrois, d'un point de vue linguistique, la dichotomie entre premier plan et arrière-plan est réalisée et identifiée à l'aide de facteurs syntaxico-sémantiques tels que la transitivité et la voix grammaticale (exemples [11b], [12]) et de facteurs fonctionnels tels que les formes référentielles et l'ordre des mots (exemples [11a], [12]). Les premiers déterminent les rôles grammaticaux et sémantiques des participants de l'action, tandis que les derniers gèrent l'organisation de l'information en attribuant les rôles de topique et de focus discursifs.

\section{Le développement de l'organisation informationnelle dans la production des enfants}

La gestion de l'organisation informationnelle requiert la maitrise de la multifonctionnalité linguistique et de la dépendance contextuelle (Hickmann, 1995; Hirzalla, 2010) ainsi que celle des calculs cognitifs simultanés (Berman et Slobin, 1994; Bowerman, 1990, cité par Berman et Slobin, 1994; Budwig et al., 2001). Ainsi, les connaissances pragmatiques générales apparaissent après l'âge de 3 ans (Schaeffer, 2000) et s'acquièrent pendant des années (Abbot Smith et al., 2001; Akhtar, 1999; MacWhinney et Bates, 1978).

Il existe peu d'études sur la capacité des enfants à gérer l'articulation discursive topique-focus prenant en compte des relations phrase-discours. Ce sont surtout les opérations de «topicalisation», ou focalisation, qui permettent de déplacer le patient de l'action à une position syntaxique plus élevée, qui ont suscité beaucoup 
d'intérêt (Berman, 1979 et 1990; Berman et Slobin, 1994; Demuth et al., 2010; Jisa et al., 2009; Jisa et al., 2010; Marchman et al., 1991; Prat-Sala et al., 2000; Schaeffer, 2000; Tomasello, 1992 et 2000; entre autres).

D'autres travaux se sont concentrés sur l'utilisation conventionnelle des expressions référentielles selon les fonctions discursives (Fekete, 2007; Hickmann et al., 1995; Jisa, 2000; Karmiloff-Smith, 1981; Vion et Colas, 1998; entre autres).

À notre connaissance, seule l'étude de Chini et Lenart (2008) a observé la gestion du topique de la phrase dans la production discursive de deux adultes, un Italien et un Français natifs, et de quelques apprenants. Toutefois, les auteurs affirment que "généralement, on ne considère pas comme topique un sujet postverbal, à moins qu'il s'agisse d'un sujet disloqué à droite, désemphatisé» et que lorsque plusieurs topiques sont possibles, la priorité pour ce rôle revient au «sujet, le topique grammaticalisé», aux formes linguistiques pronominales ou au topique du discours (Chini et Lenart, 2008 : 133). Ces critères nous laissent supposer que le topique est toujours préverbal et coïncide avec le sujet ou le topique discursif global; par conséquent, le contexte discursif n'est pas toujours pris en compte. Les résultats de l'étude citée supra sur les deux natifs montrent que le topique est généralement le protagoniste humain, qui est maintenu dans ce rôle à l'aide d'une anaphore zéro ou d'une relative en italien et d'une forme pronominale en français. Le changement de topique est généralement exprimé sous forme de syntagme nominal, dans les deux langues observées.

\section{Hypothèses}

Les données développementales sur l'articulation topique-focus étant, à notre connaissance, inexistantes, nous nous sommes fondée sur les théories mentionnées ci-dessus pour établir nos hypothèses.

Hypothèse 1: La distribution topique-focus diffère entre le discours des enfants et celui des adultes hongrois. Nous nous attendons à plus de phénomènes d'association dans tous les groupes d'âge car cette solution est plus facile d'un point de vue linguistique, pragmatique et cognitif (Berman et Slobin, 1994; Bowerman, 1990, cité par Berman et Slobin, 1994; Budwig et al., 2001; Hickmann, 1995; Hirzalla, 2010). La sélection de deux éléments différents s'avère difficile puisque l'un doit assurer la connectivité sémantique au contexte et fournir la base de la nouvelle information pendant que l'autre doit attirer l'attention de l'interlocuteur. Toutefois, le recours à la dissociation devrait augmenter progressivement avec l'âge.

Hypothèse 2: Comme les connaissances pragmatiques générales apparaissent après l'âge de 3 ans (Schaeffer, 2000) et s'acquièrent progressivement (Abbot Smith et al., 2001; Akhtar, 1999; MacWhinney et Bates, 1978), nous nous attendons à ce que les enfants relient le topique et le focus à la position initiale sans tenir compte du discours. Le groupe des plus jeunes qui se contente de faire une description des images (Berman et Slobin, 1994), change souvent le personnage en topique focalisé. Les enfants de 5 ans et 7-8 ans séparent l'à-propos et le centre d'attention des 
clauses mais ils mettent surtout l'action dans ce dernier rôle (Costa et Friedmann, 2009; MacWhinney, 1985). Les deux groupes les plus âgés séparent aisément le topique et le focus: ils mettent et gardent les personnages primaires en tant que topiques, et font varier les personnages secondaires, les inanimés et les propositions entières en position de focus, selon les besoins discursifs.

Hypothèse 3: Conformément aux règles de la langue hongroise, nous nous attendons surtout à des références morphématiques dans les fonctions de maintien et à des expressions lexicales dans les fonctions de réintroduction (Fekete, 2007). La proportion de ces moyens linguistiques augmente avec l'âge dans ces deux fonctions respectivement, et la fréquence de l'autre type de forme linguistique (l'élément lexical dans le contexte du maintien et la forme morphématique dans la reprise) devrait diminuer. L'utilisation des moyens pronominaux est insignifiante dans les fonctions de maintien et de reprise de l'association du topique-focus puisque cette forme est surtout caractéristique du contexte promu. En revanche, la fréquence des pronominaux employés pour des raisons emphatiques n'est pas importante dans le corpus et caractérise surtout les narrations des enfants plus jeunes.

L'interprétation de nos résultats prend en compte tant le développement linguistique que le développement pragmatique.

\section{Méthodologie}

\subsection{Locuteurs}

Pour étudier l'organisation de l'information dans une perspective développementale, nous avons collecté des narrations orales auprès de 75 locuteurs hongrois natifs en sélectionnant des participants de 5 groupes d'âge différents: des enfants de 3, 5, 7-8 et 11-12 ans ainsi qu'un groupe d'adultes. Chaque groupe contient 15 sujets. Les enfants allaient à la crèche, à l'école maternelle ou à l'école primaire. Les adultes étaient étudiants à l'université. Afin de garder l'anonymat des sujets et de pouvoir distinguer les textes, nous avons associé à chaque texte un code spécifique. Ce dernier stipule l'âge (année, mois) du locuteur et une lettre aléatoire (sorte de «matricule»), pour chaque groupe d'âge. Ainsi, «7;08.g» correspond au septième enfant enregistré dans le groupe des 7-8 ans et il a 7 ans et 8 mois. Le tableau 1 résume le nombre de locuteurs, leur âge moyen et l'éventail d'âges.

\begin{tabular}{|l|l|l|l|l|l|}
\hline & $\mathbf{3}$ ans & $\mathbf{5}$ ans & $\mathbf{7 - 8}$ ans & $\mathbf{1 1 - 1 2}$ ans & Adultes \\
\hline Nombre & 15 & 15 & 15 & 15 & 15 \\
\hline Éventail d’âges & $3 ; 02-3 ; 11$ & $5 ; 00-5 ; 11$ & $7 ; 01-8 ; 03$ & $11 ; 00-12 ; 01$ & $19 ; 02-29 ; 02$ \\
\hline Âge moyen & $3 ; 07$ & $5 ; 07$ & $7 ; 10$ & $11 ; 07$ & $21 ; 04$ \\
\hline
\end{tabular}

Tableau 1 - Nombre, éventail d'âges et âge moyen des sujets par groupe d'âge 


\subsection{Corpus}

Le support utilisé afin d'obtenir les données est le livre de Mayer (1969) portant le titre Frog, where are you? Il contient une histoire de 24 images sans texte, présentant les aventures de deux personnages principaux, un garçon et son chien, à la recherche de la grenouille fugueuse du garçon. Au cours de l'histoire, le garçon et le chien rencontrent des personnages secondaires: une taupe mord le nez du garçon, un essaim d'abeilles pourchasse le chien, un hibou fait tomber le garçon de l'arbre, puis le garçon reste accroché aux bois d'un cerf qui le fait tomber dans un ravin. Les deux protagonistes y retrouvent leur grenouille accompagnée d'autres grenouilles. Le garçon la récupère et ils rentrent tous les trois.

Ce livret a déjà servi de base à de nombreuses études interlinguistiques développementales travaillant sur les compétences narratives de différentes tranches d'âge (Bamberg, 1987; Berman et Slobin, 1994; Fekete, 2011; Jisa et al., 2010; Kail et Hickmann, 1992; Kern, 1997; Strömqvist et Verhoeven, 2004).

Le même protocole expérimental a été utilisé pour tous les groupes d'âges suivant les indications décrites dans Berman et Slobin (1994: 22-23). En revanche, nous avons dû apporter quelques modifications. Chaque locuteur a été interviewé individuellement dans une pièce isolée de l'institution. La procédure a été effectuée par un seul chercheur. L'introduction de la tâche était différente dans le groupe des enfants et des adultes. Chez les premiers, le chercheur a informé l'enfant de la tâche à accomplir en lui demandant de l'aide de la façon suivante: «Je viens d'acheter à ma cousine un livre qui raconte l'histoire d'un garçon, d'un chien et d'une grenouille [le chercheur a présenté chaque personnage en le pointant du doigt sur la couverture du livre]. Je ne connais pas encore l'histoire et je ne sais pas comment la lui raconter. Est-ce que tu peux m'aider?». L'indication de la méconnaissance de l'histoire par le chercheur était nécessaire pour mettre en évidence la demande d'une organisation conventionnelle de la narration : la construction avec une introduction des personnages, avec des phrases complètes, évitant les déictiques et les ellipses. Chez les adultes, cette phase de la procédure a été réduite à la simple demande de raconter l'histoire après avoir regardé les images.

Chaque narration a été enregistrée, numérisée, puis transcrite en employant le programme CLAN (Child Language Analysis). Les textes ont été fractionnés en propositions qui ont été considérées comme les plus petites unités de l'analyse. La proposition est une unité qui contient un prédicat (un verbe fléchi ou non fléchi, ou un adjectif prédicatif) exprimant une situation (activité, événement, état) (Berman et Slobin, 1986: 7).

Les analyses ont été faites à partir d'un système de codage développé pour distinguer association et dissociation du topique et/ou du focus, tous les personnages mentionnés dans ces rôles, leurs formes linguistiques et leurs fonctions discursives.

Nous distinguons quatre fonctions discursives (Jisa et Kern, 1998; Jisa, 2000). L'INTRODUCTION indique la première mention d'un personnage en topique et/ou en focus (exemple [13]). 
[13] utána meg $\quad{ }_{\mathrm{F}+\mathrm{T}} \mathrm{a}$ kis-fiú]

après et DEF petit-garçon

'et puis après, le petit garçon

[suite] meg-ij-ed-t

PV-effrayer-MOY-PAS.3S

a eu peur d'un oiseau'

[suite] utána meg [F egy szarvas] a hát-á-ra fel-vett- $\left[_{\mathrm{T}} \mathrm{e}\right]$

après et INDEF cerf DEF dos-POS-SUB PV-prendre.PAS-3SD

'et puis après, le cerf l'a pris sur son dos'

(7;08.g)

Il s'agit de MAINTIEN lorsque le même personnage est maintenu comme topique et/ou focus dans au moins deux clauses consécutives (exemple [14]).

[14] [F+T béka] nem alsz-ik grenouille NEG dormir-PRS.3S

'la grenouille ne dort pas'

[suite] ki-mász-[ $\left.{ }_{\mathrm{F}+\mathrm{T}} \mathrm{ott}\right]$

PV-grimper-PAS.3S

'[elle] est sortie'

(3;03.i)

Dans la fonction de PROMOTION, le personnage non topicalisé/non focalisé devient le topique et/ou le focus dans la clause suivante (exemple [15]).

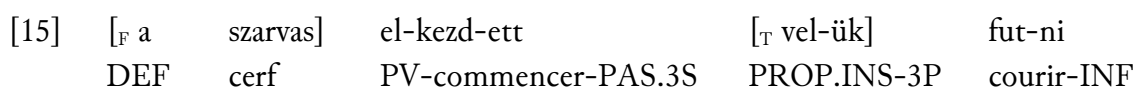

'le cerf a commencé à courir avec eux'

[suite] és [ ${ }_{\mathrm{F}}$ a kis-fiú-t $\mathbf{m e g}$ a $\quad$ kutyá-t] le-lök-t- $\left.{ }_{\mathrm{T}} \mathrm{e}\right]$

et DEF petit-garçon-ACC plus DEF chien-ACC PV-pousser-PAS-3SD

'et [il = cerf] a fait tomber le petit garçon et le chien

[suite] a pocsolyá-ba

DEF flaque-d'eau-ILL

dans la flaque d'eau'

$(11 ; 09 . h)$

La RÉINTRODUCTION indique la réintroduction d'un personnage, absent de la clause précédente, comme topique et/ou focus (exemple [16]).

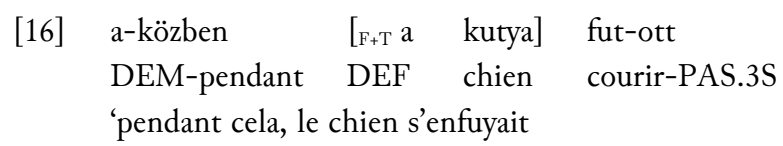




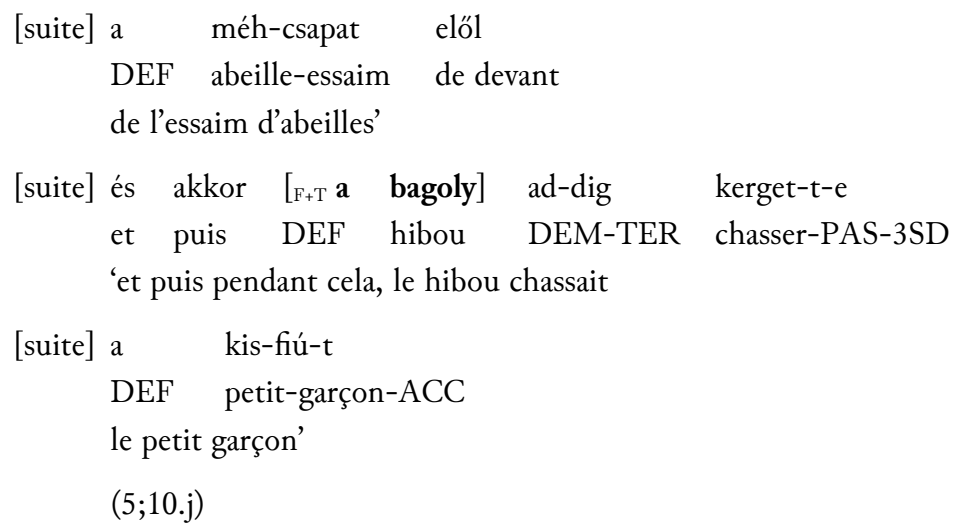

Nous avons ensuite analysé les données, entendues comme moyennes de pourcentages calculées par rapport au nombre total de clauses $(\mathrm{T})$. L'analyse de variance ANOVA, avec l'âge comme facteur principal, a été utilisée pour comparer la différence entre les groupes.

\section{Résultats}

\subsection{Association topique-focus}

Dans la présente étude, nous n'exposons que les principaux résultats pour le topique et le focus discursifs locaux en hongrois.

Le graphique 1 illustre la distribution de topique et de focus discursifs dans la narration hongroise ( $\mathrm{T}=$ nombre total de clauses des textes narratifs).

Comme l'indique ce graphique, l'association topique-focus (exemple [17]) domine dans les narrations des enfants et des adultes hongrois.

[17] közben $\quad\left[_{F+T}\right.$ a kutyá-t] jól

entre-temps DEF chien-ACC bien

[suite] meg-kerget-t-ék a darazs-ak

PV-poursuivre-PAS-3PD DEF guêpe-PL

'entre-temps, les guêpes ont bien poursuivi le chien'

[suite] mert szemtelen mód le-dob-t- $\left[F_{+} \mathbf{a}\right]$ ők-et

parce que insolent façon PV-jeter-PAS-3SD PROP3P-ACC

'parce qu' [il = chien] les a méchamment fait tomber

[suite] a fá-ról

DEF arbre-DEL

de l'arbre'

$(21 ; 05 . \mathrm{e})$ 


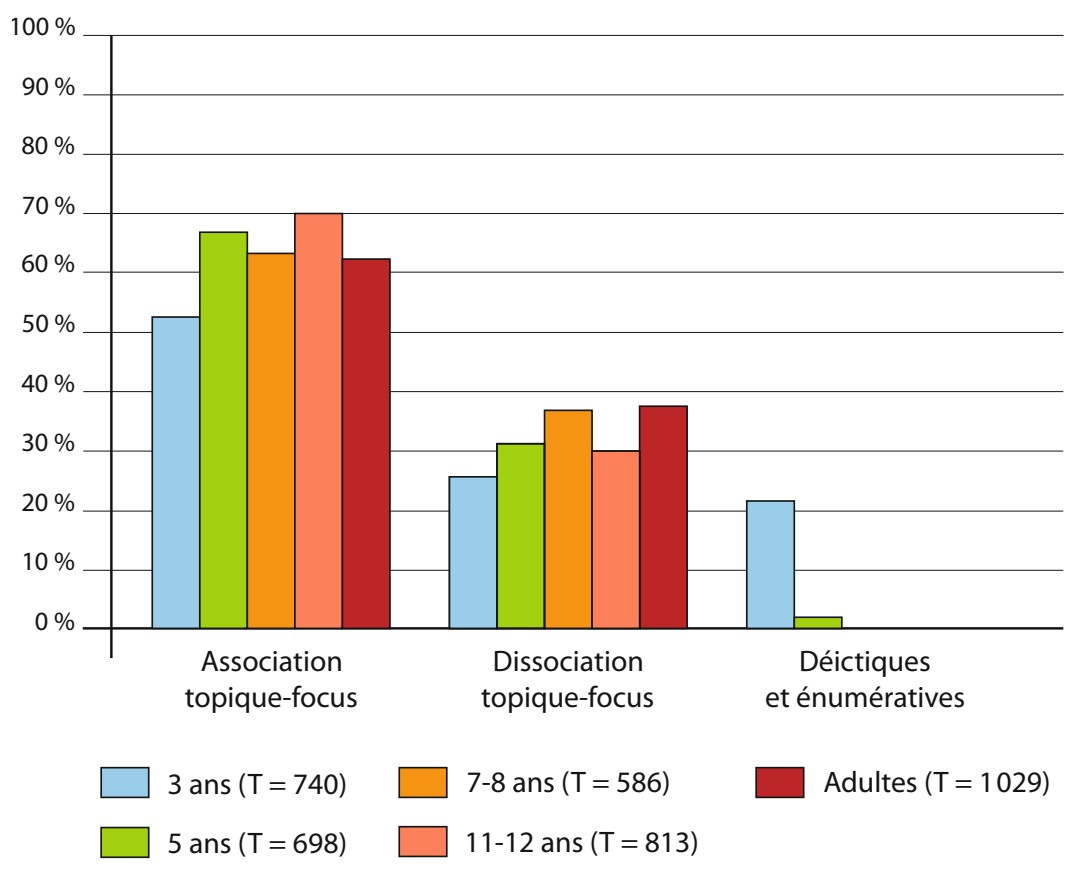

Graphique 1 - Distribution de topique discursif et de focus discursif dans la narration hongroise

Dans le cas des propositions où ni la position syntaxique ni le moyen linguistique ne permettent de déterminer le statut pragmatique des différents éléments (exemple [17]), le contexte discursif, plus précisément le statut des éléments de la proposition précédente, doit être pris en considération. En effet, si le locuteur avait voulu changer la distribution des rôles, il aurait employé des moyens linguistiques plus explicites (des formes lexicales).

Dans la deuxième proposition de l'exemple [17], le «chien» est indexé sur le verbe et les «abeilles» sont mentionnées à l'aide d'une forme pronominale. Ils remplissent donc tous le rôle de topique mais ne véhiculent pas la même saillance informationnelle (Givón, 2001). Étant donné que le «chien» apparaît en tant que topique focalisé dans la clause précédente, il garde le même statut en tant que topique primaire dans la deuxième proposition.

L'emploi séparé de ces deux rôles discursifs (exemple [18]) $\left(F_{(4,70)}=4,39, p<, 004\right)$ augmente significativement avec l'âge.
[18] aztán
$[\mathrm{F}+\mathrm{T}$ a
kutya] ki-es-ett
aZ
ablak-on
puis
DEF chien
PV-tomber-PAS.3S
$\mathrm{DEF}$
fenêtre-SUP
'puis le chien est tombé par la fenêtre' 
[suite] és ${ }_{\mathrm{F}}$ a $\quad$ kis-fiú $] \quad$ el-kap-t- $\left[_{\mathrm{T}} \mathbf{a}\right]$

et DEF petit-garçon PV-recevoir-PAS-3SD

'et le petit garçon l'a attrapé'

$(5 ; 08 . d)$

Le graphique 2 nous instruit sur les éléments choisis pour le rôle de l'association topique-focus par les locuteurs hongrois $(\mathrm{T}=$ nombre total de clauses de l'association topique-focus).

L'association est généralement attribuée aux personnages primaires (exemple [19]) dans le discours hongrois et ce dès l'âge de 3 ans.

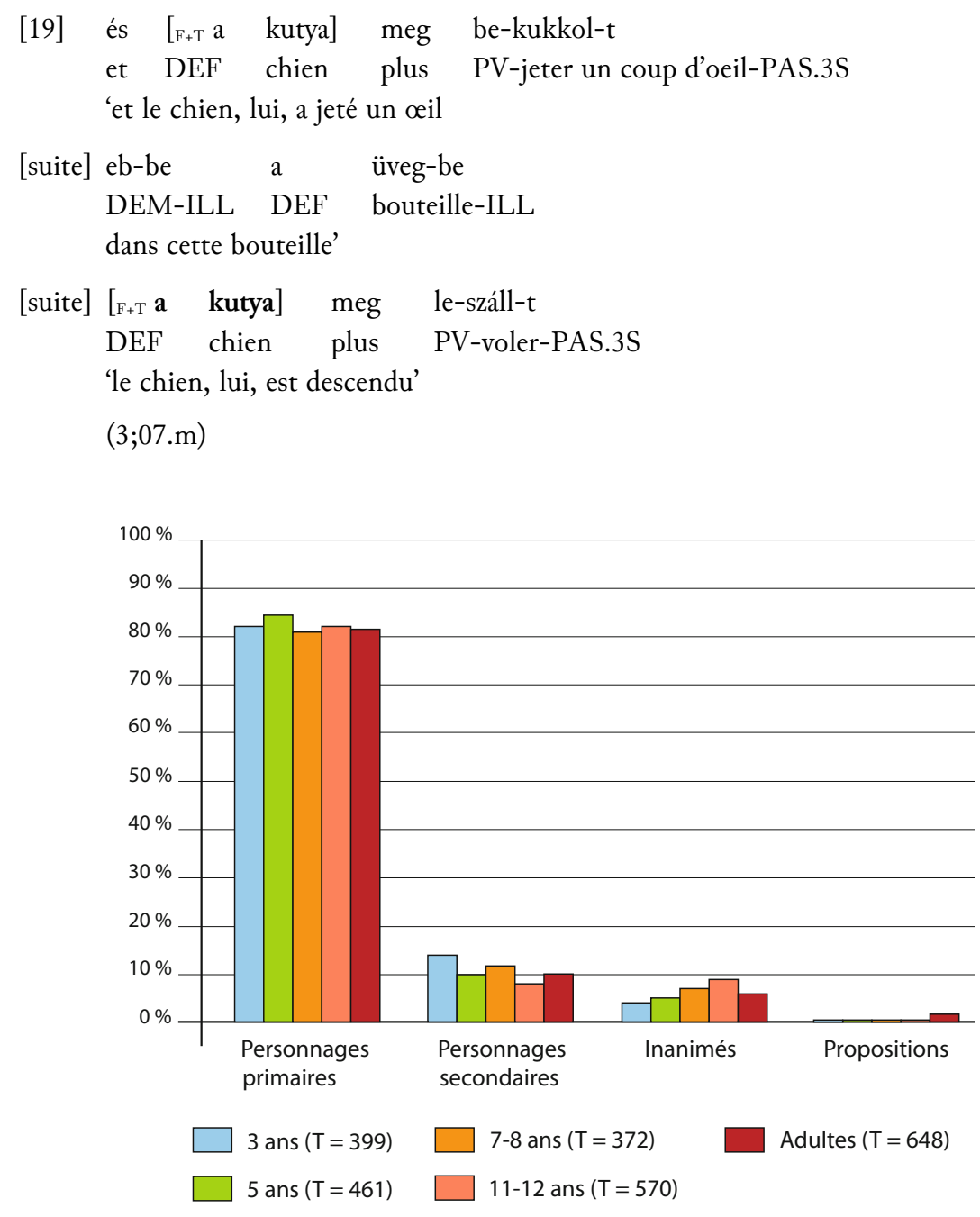

Graphique 2 - Éléments choisis pour le rôle de l'association topique-focus dans la narration hongroise 


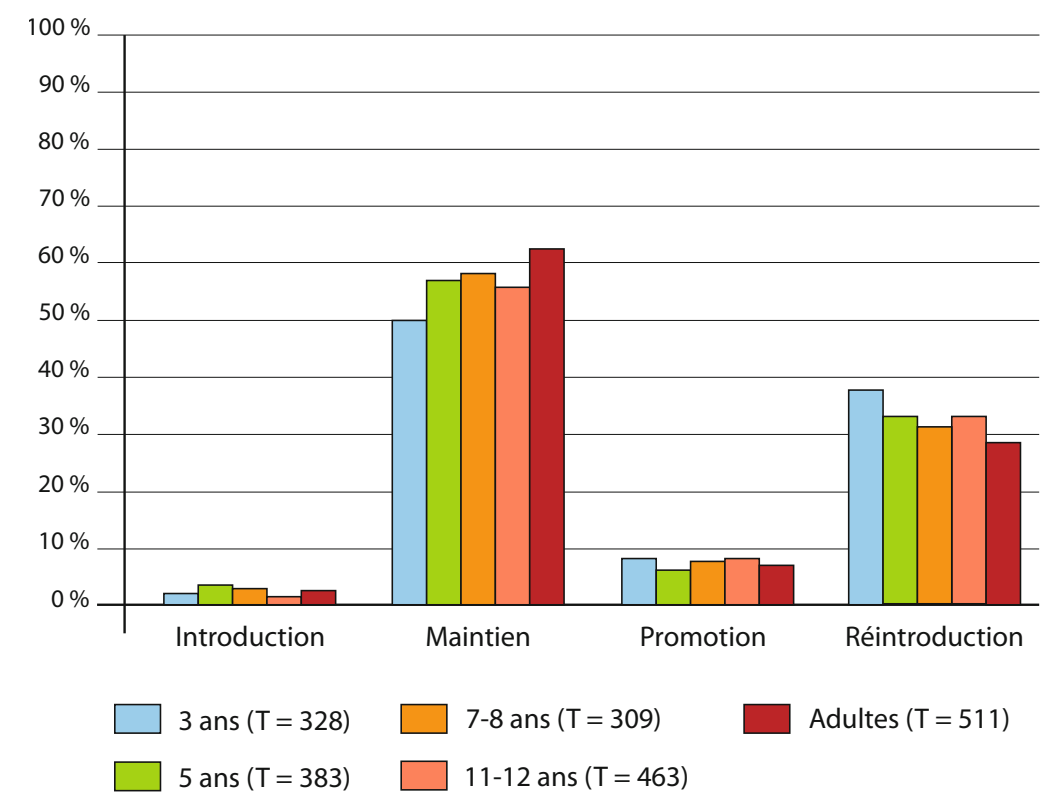

Graphique 3 - Fonctions discursives des personnages primaires comme topiques-focus dans la narration hongroise

Le graphique 3 synthétise les fonctions discursives de l'association topique-focus pour les personnages primaires dans la narration hongroise $(T=$ nombre total de clauses de l'association pour les personnages primaires).

Le maintien (exemple [20]) des personnages primaires dans ce rôle augmente avec l'âge $\left(F_{(4,70)}=3,14, p<, 02\right)$ alors que leur réintroduction (exemple [21]) diminue $\left(F_{(4,70)}=2,82, p<, 03\right)$.

[20] talál-t- $\left[{ }_{F+T}\right.$ ak $]$ egy méh-kas-t

trouver-PAS-3PG INDEF abeille-ruche-ACC

'[ils = garçon + chien $]$ ont trouvé une ruche d'abeilles'

[suite] és $\quad\left[_{\mathrm{F}_{\mathrm{T}} \mathrm{T}}\right.$ kis-fiú] bele-kiabál-t a lyuk-ba

et DEF petit-garçon PV-crier-PAS.3S DEF trou-ILL

'et le petit garçon a crié dans le trou'

$(7 ; 01 . d)$

[21] itt meg $\mathrm{FF}_{+\mathrm{T}}$ a szarvas] fut-ott

ici plus DEF cerf courir-PAS.3S

'et ici, le cerf courait' 
[suite] itt meg le-es- $\left[_{\mathrm{F}+\mathrm{T}}\right.$ ett $]$ a tó-ba

ici plus PV-tomber-PAS.3S DEF lac-ILL

'et ici, [il = garçon] est tombé dans le lac'

$(3 ; 08 . n)$

En ce qui concerne les moyens linguistiques, le graphique 4 illustre ceux qui sont utilisés pour référer aux personnages primaires maintenus comme topiques-focus dans la narration hongroise $(T=$ nombre total de clauses avec des personnages principaux maintenus comme topiques-focus).

Les protagonistes sont généralement maintenus en tant que topiques-focus sous forme suffixale (exemple [22]) dans tous les groupes d'âge.
és akkor megint
ott
oda-mász-[F+T ott]
a hát-á-ra
et puis de nouveau là-bas PV-grimper-PAS.3S DEF dos-POSS-SUB

'et puis là-bas, [il = garçon] a de nouveau grimpé sur son dos [= cerf]'
[suite] és akkor le-es- ${ }_{\mathrm{F}+\mathrm{T}}$ ett] a tó-ba
et puis PV-tomber-PAS.3S DEF lac-ILL
'et puis [il = garçon] est tombé dans le lac'

$(3 ; 02 . d)$

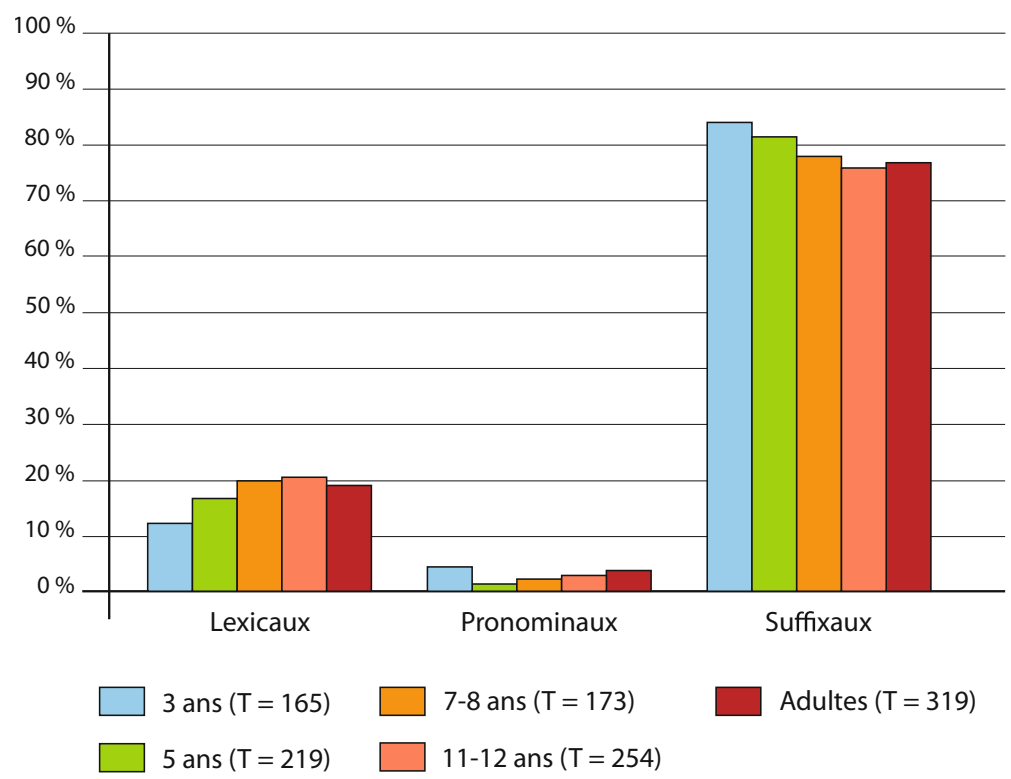

Graphique 4 - Moyens linguistiques pour les personnages primaires maintenus comme topiques-focus dans la narration hongroise 


\subsection{Topique dissocié}

Les résultats montrent des similitudes entre le rôle de topique associé et celui de topique dissocié. Le graphique 5 résume les types d'entités sélectionnés par les locuteurs hongrois pour le rôle de topique dissocié $(\mathrm{T}=$ nombre total de clauses contenant un topique dissocié).

Ce graphique signale une préférence pour les personnages primaires (exemple [23]) dans la fonction de topique dissocié, similairement au topique associé, dans tous les groupes d'âge.

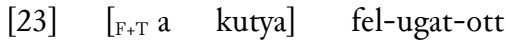 \\ DEF chien PV-aboyer-PAS.3S}

'le chien a aboyé un coup'

$\begin{array}{rlllll}\text { [suite] de } & { }_{\mathrm{F}+\mathrm{T}} \text { a } & \text { méh-ek] } & \text { egyre csak } & \text { kerget-t-ék } & \left.{ }_{\mathrm{T}} \text { ök-et }\right] \\ \text { mais } & \mathrm{DEF} & \text { abeille-P } & \text { sans arrêt } & \text { poursuivre-PAS-3PD } & \text { PP3P-ACC }\end{array}$

'mais les abeilles ne cessaient pas de les poursuivre'

$(5 ; 08 . e)$

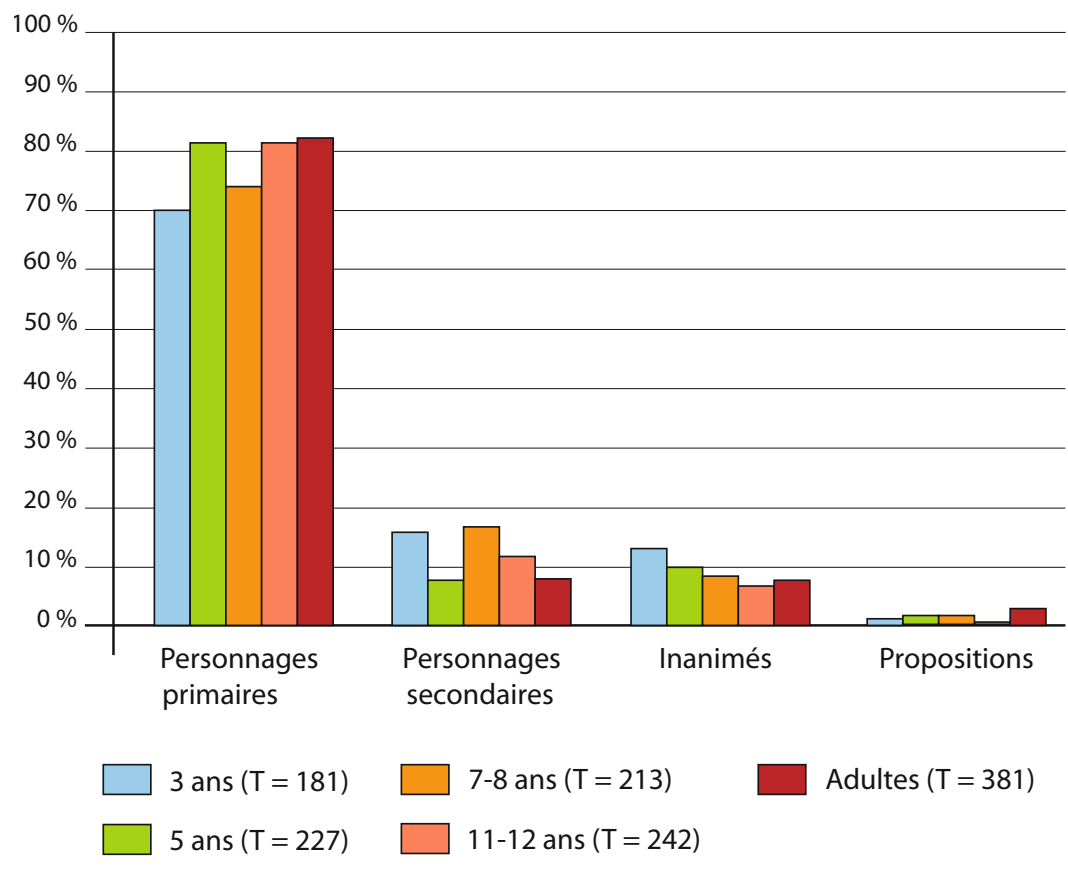

Graphique 5 - Éléments choisis pour le rôle du topique dissocié dans la narration hongroise 


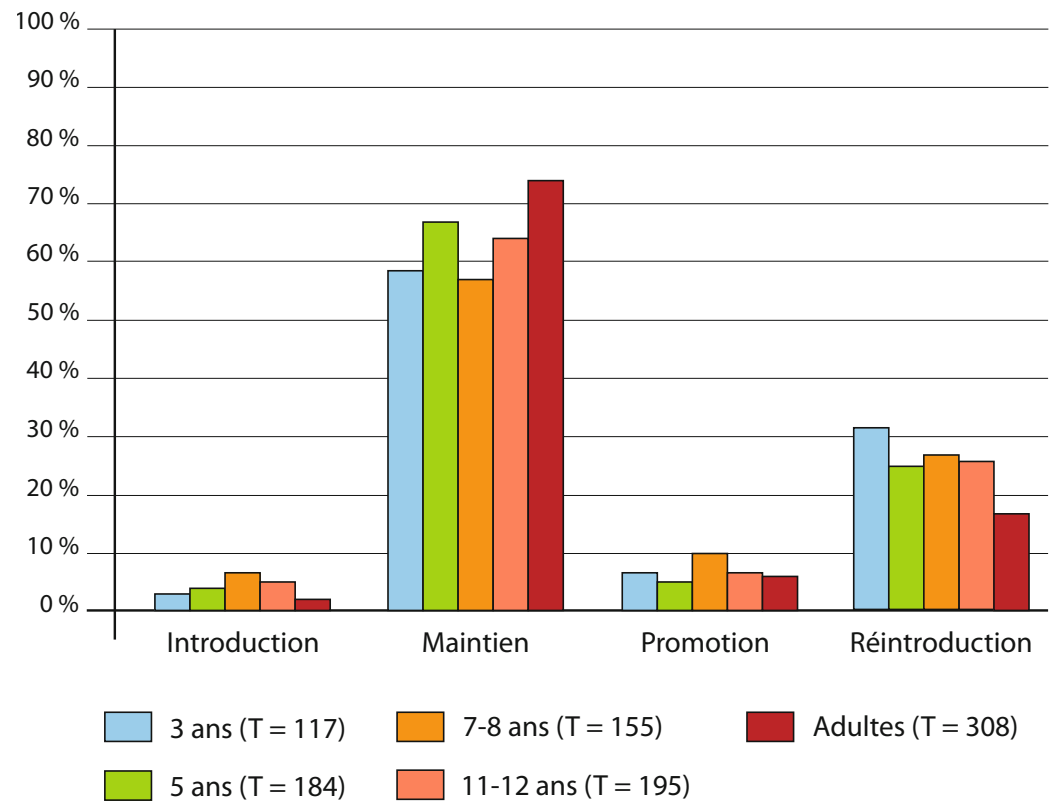

Graphique 6 - Fonctions discursives des personnages primaires remplissant le rôle du topique dissocié dans la narration hongroise

Le graphique 6 illustre les fonctions discursives des personnages principaux topiques $(\mathrm{T}=$ nombre total de clauses avec des personnages principaux comme topiques dissociés).

Tous les groupes d'âge préfèrent maintenir (exemple [24]) les personnages primaires dans ce rôle. Cette tendance augmente avec l'âge $\left(F_{(4,70)}=3,14, p<, 02\right)$ alors que leur réintroduction (exemple [25]) diminue $\left(F_{(4,70)}=2,63, p<, 05\right)$.

[24] utána meg ${ }_{\mathrm{F}+\mathrm{T}}$ a $\quad$ kis-fiú]

après et DEF petit-garçon

'et puis après, le petit garçon

[suite] meg-ij-ed-t

egy

madár-tól

PV-effrayer-MOY-PAS.3S INDEF

oiseau-ABL

a eu peur d'un oiseau'

[suite] utána meg [F egy szarvas] a hát-á-ra fel-vett- $\left[_{\mathrm{T}} \mathbf{e}\right]$

après et DEF cerf DEF dos-POS-SUB PV-prendre.PAS-3SD

'et puis après, le cerf l'a pris sur son dos'

$(7 ; 08 . g)$ 
[25] [Déict ott a kicsi kecske]

là-bas DEF petit chèvre

'là-bas la petite chèvre'

[suite] és [F víz-be] [T van]

et eau-ILL être.PRS.3S

'et dans de l'eau, [il = garçon] y est'

$(3 ; 03 . i)$

Le graphique 7 nous instruit sur les formes linguistiques attestées pour les personnages primaires maintenus comme topiques dans la narration hongroise ( $\mathrm{T}=$ nombre total de clauses contenant des personnages principaux maintenus en tant que topiques).

Les protagonistes sont généralement maintenus avec des suffixes verbaux (exemple [26]). Les enfants emploient souvent des moyens lexicaux (exemple [27]) jusqu'à l'âge de 7-8 ans mais ces derniers diminuent de façon significative $\left(F_{(4,70)}=\right.$ $6,51, p=, 0002)$ dans les deux groupes les plus âgés.

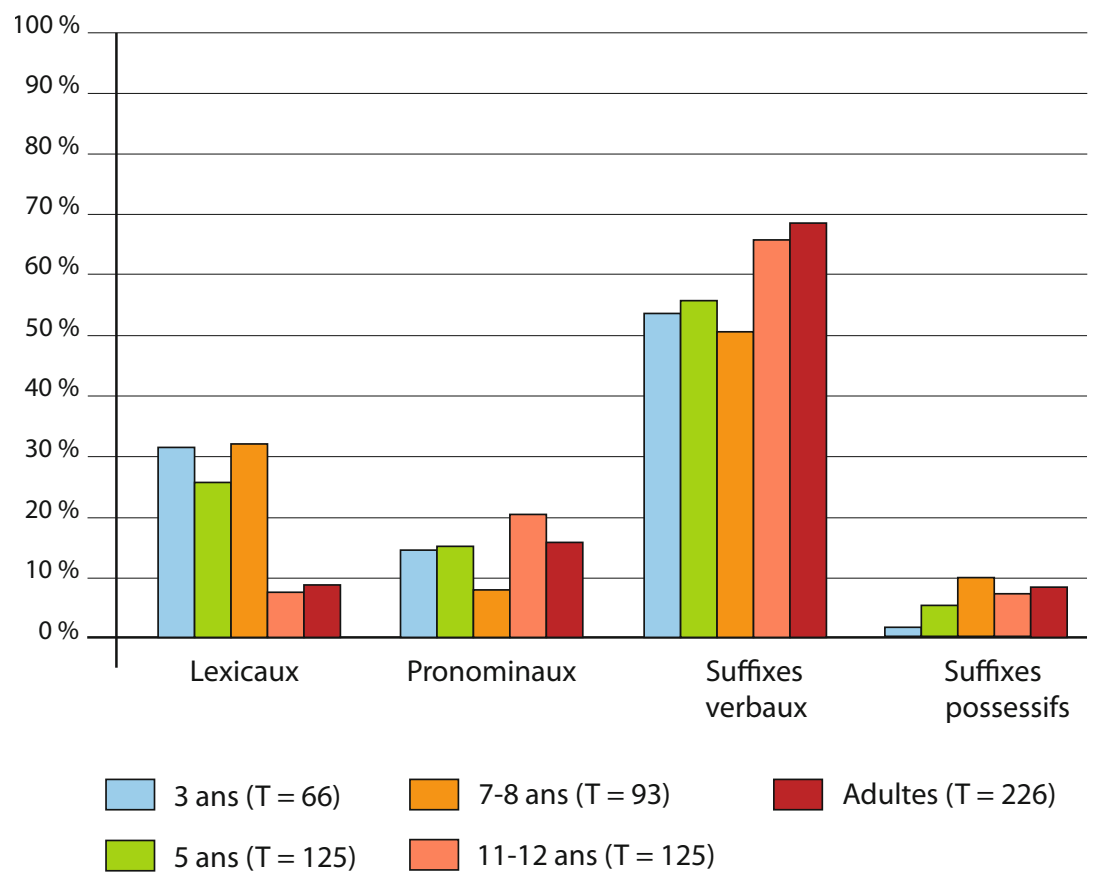

Graphique 7 - Moyens linguistiques pour les personnages primaires maintenus comme topiques dans la narration hongroise 


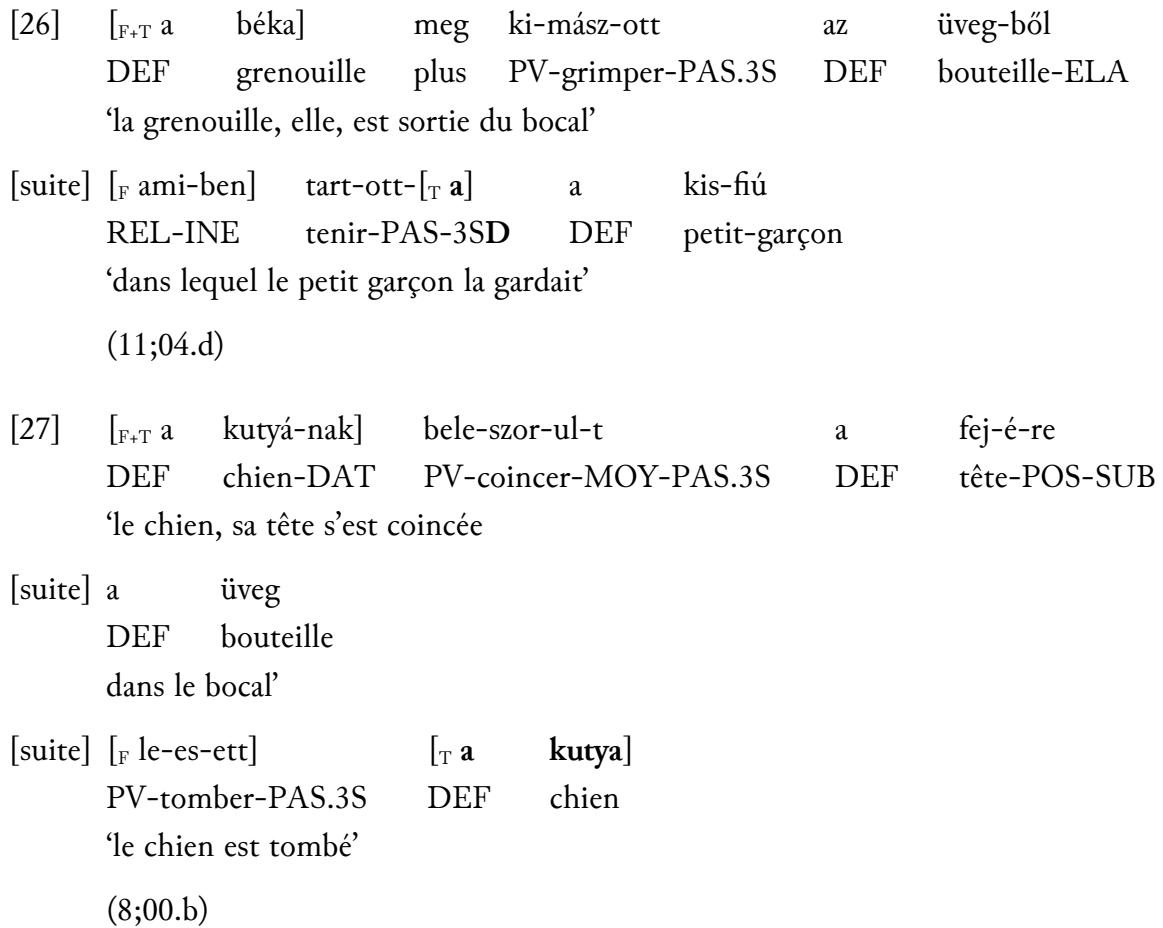

\subsection{Focus dissocié}

Concernant le focus dissocié, c'est dans la sélection des éléments assurant ce rôle qu'on observe le plus de diversité du point de vue développemental. Le graphique 8 donne des renseignements sur la répartition des personnages et des entités inanimées attestés en tant que focus dissociés dans les textes des enfants et des adultes hongrois ( $\mathrm{T}=$ nombre total de clauses avec un focus dissocié) .

Les enfants hongrois préfèrent y mettre le verbe (exemple [28]). Cette tendance diminue $\left(F_{(4,70)}=7,01, p<, 0001\right)$ à partir de $7-8$ ans.
[28] [F bele-kiabál-t $] \quad{ }_{\mathrm{T}}$ a $\quad$ kutya $]$
PV-crier-PAS.3S DEF chien
'et le chien a crié dedans'

$\begin{array}{rllll}\text { [suite] és } & \left.{ }_{\mathrm{F}} \text { üldöz-ni }\right] & \left.\text { kezd-t- }{ }_{\mathrm{T}} \text { ék }\right] & \text { a } & \text { méh-ek } \\ \text { et } & \text { poursuivre-INF } & \text { commencer-PA-3PD } & \text { DEF } & \text { abeille-P }\end{array}$

'et les abeilles ont commencé à $[\mathrm{le}=$ chien] poursuivre'

$(5 ; 07 . b)$

Les enfants de 11-12 ans et les adultes placent surtout des inanimés (exemple [29]) en focus. Ceux-ci sont attestés majoritairement dans la fonction d'introduction et sous forme d'oblique. 


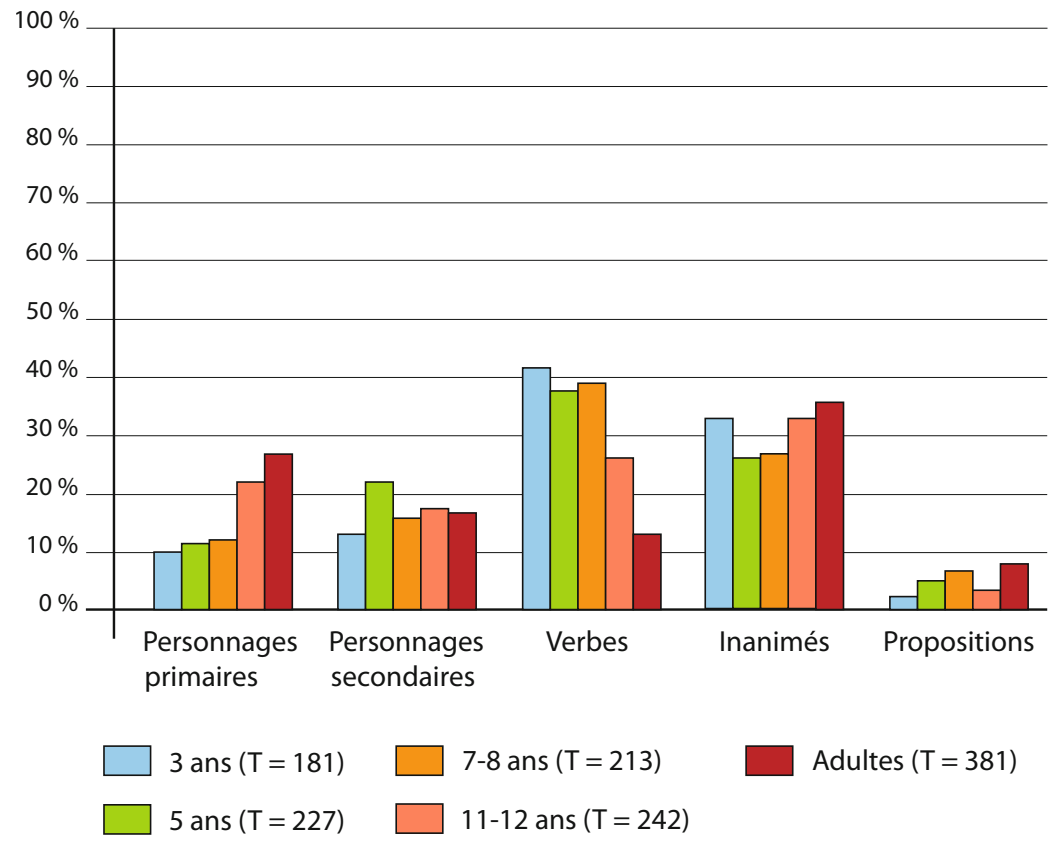

Graphique 8 - Éléments choisis pour le rôle de focus dissocié dans la narration hongroise

[29] aztán ment- $\left.{ }_{\mathrm{F}+\mathrm{T}} \mathrm{ek}\right]$ kifelé puis aller.PAS-3P vers l'extérieur

'puis [ils = garçon + chien] allaient dehors'

[suite] ${ }_{\mathrm{F}}$ az ablak-ból] kiabál-t $\quad{ }_{\mathrm{T}}$ Peti]

DEF fenêtre-ELA crier-PAS.3S Peti

'Peti criait de la fenêtre'

$(11 ; 00 . \mathrm{m})$

La focalisation sur les personnages primaires (exemple [30]) augmente $\left(F_{(4,70)}=\right.$ $7,03, p<, 0001)$ dans les deux groupes les plus âgés. Les locuteurs s'en servent généralement pour réintroduire les protagonistes.
[30] há
[F+T a szarvas] éppen
fel-emel-i
donc DEF cerf être en train de
PV-lever-PRS.3SD
'donc le cerf est juste en train de soulever
[suite] a fej-é-t
DEF tête-POS-ACC
sa tête'




\begin{tabular}{|c|c|c|c|c|}
\hline [suite] ${ }_{\mathrm{F}} \mathbf{a}$ & kis-gyerek] & fel-akad & {$\left[{ }_{\mathrm{T}} \mathrm{a}\right.$} & $\begin{array}{l}\text { fej-é-re] } \\
\text { tête-POS-SUB }\end{array}$ \\
\hline DEF & petit-enfant & PV-rester accroché.PRS.3S & DEF & \\
\hline
\end{tabular}

$(29 ; 02.0)$

\section{Discussion}

Dans cette étude, notre objectif était de clarifier les notions de topique et de focus, puis d'observer la trajectoire développementale du topique et du focus discursifs locaux en hongrois.

Nous avons proposé une définition opérationnelle du topique et du focus discursifs locaux en hongrois: la dichotomie entre premier plan et arrière-plan est réalisée linguistiquement par le locuteur et identifiée par l'interlocuteur grâce aux facteurs syntaxico-sémantiques (transitivité, voix) et fonctionnels (formes référentielles, ordre des mots) en hongrois. Tandis que les premiers déterminent les rôles grammaticaux et sémantiques des participants de l'action, les derniers gèrent l'organisation de l'information en attribuant les rôles de topique et de focus discursifs.

Nous souscrivons au point de vue largement partagé suivant lequel le topique discursif identifie l'item linguistique à propos duquel une nouvelle information est apportée dans la clause. Sa fonction consiste à déterminer la perspective suivant laquelle l'information est présentée et à connecter cette information au contexte (Cornish, 2004). Il fait partie de l'arrière-plan et sert de point d'ancrage sémantique dans le discours. Il est généralement pronominalisé ou indexé sur le verbe dans des contextes de maintien (Gécseg et Kiefer, 2009; Lambrecht, 1994 ; Goldberg, 2004; Neeleman et al., 2009; Tolcsvai Nagy, 2001; Van Rooij et Schulz, 2017) et exprimé par un syntagme nominal dans des contextes de changement. Le terme placé en focus discursif véhicule l'information la plus significative du point de vue sémantique, indépendamment de son degré d'accessibilité, et se situe au centre d'attention du locuteur et de l'interlocuteur (Dik, 1997; Givón, 1988 ; Hajičová et Sgall, 1984; Hickmann, 1995; Likhacheva-Philippe, 2010 ; Tolcsvai Nagy, 2001; Stutterheim et Klein, 1989). Il peut véhiculer une information nouvelle ou ancienne. La forme linguistique choisie pour l'expression du focus dépend de la présence de cet item dans le contexte discursif. Lorsque le focus comporte une information connue ou déductible du contexte précédent, les formes référentielles (zéro et pronominales) sont favorisées. Lorsqu'on a affaire à une information nouvelle, pas encore évoquée, ce sont les formes nominales qui servent à l'encodage linguistique du focus discursif. L'ordre des mots étant déterminé par leur saillance informationnelle (Enkvist, 1989; Gernsbacher, 1995 ; Givón, 2001; Likhacheva-Philippe, 2010; Virtanen, 2004), nous suggérons qu'en hongrois, le focus discursif est toujours placé en position initiale de la clause, ce 
qui n'est pas le cas du topique discursif (Gécseg et Kiefer, 2009; Givón, 2001; Lambrecht, 1994; Neeleman et al., 2009; Tolcsvai Nagy, 2006). Ce dernier s'y trouve lorsqu'il est focalisé.

Ce phénomène aboutit, dans les textes hongrois, à des associations topique-focus (quand le topique est promu et donc focalisé en position initiale de la clause) et des dissociations (quand l'à-propos et l'élément véhiculant la saillance informationnelle sont des arguments différents).

Nous avons étudié les personnages mentionnés, leurs formes linguistiques et leurs fonctions discursives en distinguant deux possibilités, à savoir les associations et les dissociations topique-focus.

Nos principaux résultats confirment le consensus général suivant lequel les locuteurs préfèrent promouvoir le topique à la position initiale dans les textes narratifs, conformément à notre hypothèse 1 . En revanche, la dissociation entre les deux possibilités augmente avec l'âge, ce qui soutient également notre hypothèse 1 . Même les enfants les plus jeunes sont capables de dissocier les deux rôles, ce qui représente $20 \%$ de leur production. Ce chiffre se double chez les adultes. Les topiques dissocié et associé sont utilisés pour le maintien des personnages récurrents à l'aide de suffixes verbaux, ce que prédisent nos hypothèses 2 et 3 , rejoignant les résultats français et italiens de Chini et Lenart (2008). Les enfants attirent l'attention surtout sur les actions jusqu'à l'âge de 7-8 ans (Costa et Friedmann, 2009; MacWhinney, 1985), tandis que les adolescents et les adultes recourent au rôle de focus dissocié pour introduire des entités inanimées et pour réintroduire les personnages primaires, ce qui confirme notre hypothèse 2 .

Les résultats observés dans les textes narratifs en hongrois montrent que la capacité à organiser et à hiérarchiser l'information, activité qui exige de la flexibilité cognitive chez le locuteur, se développe progressivement (Abbot Smith et al., 2001; Akhtar, 1999; MacWhinney et Bates, 1978). Toutefois, les enfants sont très tôt, dès l'âge de 3 ans, sensibles à la propriété multifonctionnelle de la langue, notamment à la possibilité de l'encodage simultané des fonctions phrastiques et discursives des formes linguistiques.

Pour les enfants, le topique focalisé est linguistiquement et cognitivement plus facile à gérer que la dissociation, car ces constructions demandent moins de calculs simultanés (Berman et Slobin, 1994; Bowerman, 1990; Budwig et al., 2001; Hickmann, 1995; Hirzalla, 2010) que les clauses avec une dissociation topique-focus. Ces dernières demandent une implication importante des facteurs fonctionnels (de l'ordre des mots et des formes référentielles) qui agissent au niveau du discours.

L'analyse des formes linguistiques utilisées pour l'expression de différentes fonctions discursives indique que, dans la narration en hongrois, l'éventail des fonctions et celui des formes linguistiques sont similaires chez l'enfant dès l'âge de 3 ans et chez l'adulte. C'est surtout l'association conventionnelle des formes linguistiques aux fonctions discursives qui pose des difficultés aux enfants. 


\section{Références bibliographiques}

Аввот-Smith, K., Lieven, E. et Tomasello, M. 2001. What Preschool Children Do and Do Not Do with Ungrammatical Word Orders. Cognitive Development 16 (2) : 679-692.

Aкнtar, N. 1999. Acquiring Basic Word Order: Evidence for Data-Driven Learning of Syntactic Structure. Journal of Child Language 26 (2) : 339-356.

Bamberg, M. 1987. The Acquisition of Narratives: Learning to Use Language. Berlin New York - Amsterdam : De Gruyter.

Berman, R. A. 1979. Form and Function: Passives, Middles, and Impersonals in Modern Hebrew. Proceedings of the Annual Meeting of the Berkeley Linguistics Society 5: 1-39. En ligne à l'adresse suivante: https://journals.linguisticsociety.org/proceedings/index. php/BLS/article/download/3248/2937.

Berman, R. A. 1990. On Acquiring (S)VO Language: Subjectless Sentences in Children's Hebrew. Linguistics 28 (6): 1135-1166.

Berman, R. A. et Slobin, D. I. 1986. Frog Story Procedures in Coding Manual: Temporality in Discourse. Berkeley: University of California, Institute of Human Development.

Berman, R. A. et Slobin, D. I. (éd.) 1994. Relating Events in Narrative. A Crosslinguistic Developmental Study. Hillsdale: L. Erlbaum.

Bowerman, M. 1990. When a Patient Is the Subject: Sorting out Passives, Anticausatives, and Middles in the Acquisition of English. In Symposium on Voice (University of California, Santa Barbara, March 8-11, 1990).

Budwig, N., Stein, S. et O’Brien, C. 2001. Non-Agent Subjects in Early Child Language: A Crosslinguistic Comparison. In K. E. Nelson, A. Aksu-Koç et C. E. Johnson (éd.), Children's Language. Mahwah: L. Erlbaum. Vol. 11: Interactional Contributions to Language Development: 49-67.

Chafe, W. L. 1987. Cognitive Constraints on Information Flow. In R. S. Tomlin (éd.), Coberence and Grounding in Discourse. Amsterdam: J. Benjamins: 21-51.

Chini, M. et Lenart, E. 2008. Identifier le topique dans une tâche narrative en italien et en français chez les natifs (L1) et les apprenants (L2). Acquisition et interaction en langue étrangère 26 : 129-148. En ligne à l'adresse suivante: http://journals.openedition. org/aile/3302.

Coppock, E. et Wechsler, S. 2012. The Objective Conjugation in Hungarian : Agreement without Phi-Features. Natural Language and Linguistic Theory 30 (3): 699-740.

Cornish, F. 2004. "Focus of Attention" in Discourse: A Comparison between the Four FG Topic Functions and the Systems of "Focus" and "Deixis" in the Columbia School of Linguistics. In J. L. Mackenzie et M. A. Gómez-GonzÁLez (éd.), A New Arcbitecture for Functional Grammar. Berlin - New York: De Gruyter: 117-150.

Costa, J. et Friedmann, N. 2009. Hebrew and Arabic Children Going Romance: On the Acquisition of Word Order in Semitic and Romance. In E. O. Авон, E. VAN DER Linden, J. Quer et P. SleEman (éd.), Romance Languages and Linguistic Theory. Selected Papers from “Going Romance” Amsterdam 2007. Amsterdam: J. Benjamins : 51-66.

Creissels, D. 2006. Syntaxe générale: une introduction typologique. Paris : Hermes ScienceLavoisier [2 t.]. 
Demuth, K., Moloi, F. et Machobane, M. 2010. 3-Year-Olds' Comprehension, Production, and Generalization of Sesotho Passives. Cognition 115 (2): 238-251.

Den Diknen, M. 2006. When Hungarians Agree (to Disagree): The Fine Art of "Phi" and “Art”. New York: CUNY Graduate Center.

Dik, S. C. 1997. Pragmatic Functions. In K. Hengeveld (éd.), The Theory of Functional Grammar. Berlin - New York: De Gruyter. Part. I: The Structure of the Clause: 309-338 [2 éd.].

Enkvist, N. E. 1989. Connexity, Interpretability, Universes of Discourse, and Text Worlds. In S. Allén (éd.), Possible Worlds in Humanities, Arts and Sciences. Berlin - New York: De Gruyter: 162-186.

Fekete, G. 2007. Referential Cohesion in Hungarian: A Developmental Study. In Proceedings of the Second Oxford Postgraduate Conference in Linguistics - LingO-2007. 75-82. En ligne à l'adresse suivante: http://citeseerx.ist.psu.edu/viewdoc/download?doi=10.1.1.3 63.3082\& 2 rep $=$ rep $1 \&$ type $=$ pdf.

FeKete, G. 2011. Manipulation de l'organisation informationnelle: étude développementale de la narration en hongrois. Thèse de doctorat. Université Lumière - Lyon 2.

Gabelentz, H. G. C. von DER 1869. Ideen zu einer vergleichenden Syntax. Wort- und Satzstellung. Zeitschrift für Völkerpsychologie und Sprachwissenschaft 6: 376-384.

Galmiche, M. 1992. Au carrefour des malentendus: le thème. L'information grammaticale $54: 3-10$

GÉcseg, Z. et Kiefer, F. 2009. A New Look at Information Structure in Hungarian. Natural Language and Linguistic Theory 27 (3) : 583-622.

Gernsbacher, M. A. 1995. The Structure Building Framework: What It Is, What It Might Also Be, and Why. In B. K. Britton et A. C. Graesser (éd.), Models of Text Understanding. Mahwah: L. Erlbaum: 289-311.

Givón, T. 1988. The Pragmatics of Word Order: Predictability, Importance and Attention. In M. Hammond, E. A. Moravcsik et J. R. Wirth (éd.), Studies in Syntactic Typology. Amsterdam - Philadelphie: J. Benjamins: 243-284.

Givón, T. 1992. The Grammar of Referential Coherence as Mental Processing Instructions. Linguistics 30 (1): 5-55.

Givón, T. 2001. Syntax: An Introduction. Amsterdam - Philadelphie: J. Benjamins. Vol. II.

Goldberg, A. E. 2004. Pragmatics and Argument Structure. In L. R. Horn et G. WARD (éd.), The Handbook of Pragmatics. Oxford: Blackwell: 427-441.

Grobet, A. 2002. L'identification des topiques dans les dialogues. Bruxelles - Paris: Duculot.

Gundel, J. K. et Fretheim, T. 2004. Topic and Focus. In L. R. Horn et G. Ward (éd.), The Handbook of Pragmatics. Oxford: Blackwell: 174-196.

HajIČová, E. 2007. Information Structure from the Point of View of the Relation of Function and Form. The Prague Bulletin of Mathematical Linguistics 88: 53-72.

Hajıčová, E. et Sgall, P. 1984. Text-and-Inference Based Answering of Questions. In P. Sgall (éd.), Contributions to Functional Syntax, Semantics, and Language Comprehension. Amsterdam - Philadelphie: J. Benjamins: 291-320. 
Hickmann, M. 1995. Discourse Organization and the Development of Reference to Person, Space, and Time. In P. Fletcher et B. MacWhinney (éd.), The Handbook of Child Language. Malden - Oxford : Blackwell : 194-218.

Hickmann, M., Kail, M. et Roland, F. 1995. Cohesive Anaphoric Relations in French Children's Narratives as a Function of Mutual Knowledge. First Language 15 (45) : 277-300.

Hirzalla, H. 2010. Discourse Construction at 4-5 Years of Age: Language Specificities and "Cognitive Functional" Constraints. California Linguistic Notes 35 (2): 1-31. En ligne à l'adresse suivante: http://english.fullerton.edu/publications/clnArchives/pdf/ Hirzalla-DscCnstr.pdf.

JisA, H. 2000. Increasing Cohesion in Narratives: A Developmental Study of Maintaining and Reintroducing Subjects in French. Linguistics 38 (3): 591-620. En ligne à l'adresse suivante : http://www.ddl.cnrs.fr/fulltext/jisa/Jisa_2000c.pdf.

Jisa, H., Chenu, F., Fekete, G. et Omar, H. 2010. Promoting Patients in Narrative Discourse: A Developmental Perspective. In M. Kail et M. Hickmann (éd.), Language Acquisition across Linguistic and Cognitive Systems. Amsterdam - Philadelphie: J. Benjamins: 161-177.

Jisa, H., Chenu, F., Fekete, G., Omar, H. et Saïdi, D. 2009. Promouvoir les patients dans le discours narratif: une perspective développementale. In M. KaIL, M. FAYOL et M. Hickmann (éd.), Apprentissage des langues premières et secondes. Paris: CNRS Éditions: $179-197$.

JisA, H. et Kern, S. 1998. Relative Clauses in French Children's Narrative Texts. Journal of Child Language 25 (3) : 623-652.

Jisa, H., Reilly, J. S., Verhoeven, L., Baruch, E. et Rosado, E. 2002. Passive Voice Constructions in Written Texts: A Cross-Linguistic Developmental Study. Written Language and Literacy 5 (2): 163-181.

Kail, M. et Hickmann, M. 1992. French Children's Ability to Introduce Referents in Narratives as a Function of Mutual Knowledge. First Language 12 (34): 73-94.

Karmiloff-Smith, A. 1981. The Grammatical Marking of Thematic Structure in the Development of Language Production. In W. Deutsch (éd.), The Child's Construction of Language. Londres: Academic Press: 121-147.

KeRN, S. 1997. Comment les enfants jonglent avec les contraintes communicationnelles, discursives et linguistiques dans la production d'une narration. Thèse de doctorat en linguistique. Université Lumière - Lyon 2.

Kiefer, F. 1987. The Cases of Hungarian Nouns. Acta Linguistica Academiae Scientiarum Hungaricae 37 (1-4) : 93-101.

Kiefer, F. et Kiss, K. É. (éd.) 1994. Syntax and Semantics: The Syntactic Structure of Hungarian 27. New York - San Diego: Academic Press.

KIss, K. É. 1998. Identificational Focus versus Information Focus. Language 74 (2) : 245-273.

KIss, K. É. 2002. Eseményszerkezet és topik-predikátum tagolás a magyar mondatban. Nyelvtudományi Közlemények 101: 160-172.

KIss, K. É. 2007. Topic and Focus: Two Structural Positions Associated with Logical Functions in the Left Periphery of the Hungarian Sentence. Interdisciplinary Studies on Information Structure 6: 69-81. En ligne à l'adresse suivante: https://publishup. uni-potsdam.de/opus4-ubp/files/1430/ISIS06.pdf. 
KIss, K. É. 2008. Topic and Focus: Two Structural Positions Associated with Logical Functions in the Left Periphery of the Hungarian Sentence. Acta Linguistica Hungarica $55(3-4): 287-296$.

Kiss, K. É., KIEFER, F. et SiptÁR, P. 2003. Ujj magyar nyelvtan. Osiris Tankönyvek. Budapest: Osiris Kiadó.

Lambrecht, K. 1994. Information Structure and Sentence Form: Topic, Focus, and the Mental Representation of Discourse Referents. Cambridge: Cambridge University Press.

Lambrecht, K. 2000. When Subjects Behave Like Objects: An Analysis of the Merging of $\mathrm{S}$ and $\mathrm{O}$ in Sentence-Focus Constructions across Languages. Studies in Language 24 (3) : 611-682.

Likhacheva-Philippe, L. 2010. Problèmes de la notion de topique. La linguistique 46 (2): 127-144.

López, L. 2009. A Derivational Syntax for Information Structure. Oxford: Oxford University Press.

MacWhinney, B. 1985. Hungarian Language Acquisition as an Exemplification of a General Model of Grammatical Development. In D. I. Slobin (éd.), The Crosslinguistic Study of Language Acquisition. Hillsdale: L. Erlbaum. Vol. 2: Theoretical Issues: 1069-1155.

MacWhinney, B. et Bates, E. 1978. Sentential Devices for Conveying Givenness and Newness: A Cross-Cultural Developmental Study. Journal of Verbal Learning and Verbal Behavior 17 (5) : 539-558.

Marchman, V. A., Bates, E., Burkardt, A. et Good, A. B. 1991. Functional Constraints of the Acquisition of the Passive: Toward a Model of the Competence to Perform. First Language 11 (31): 65-92.

Mayer, M. 1969. Frog, Where Are You? New York: Dial Books for Young Readers.

MoNDADA, L. 1994. Verbalisation de l'espace et fabrication du savoir: approche linguistique de la construction des objets de discours. Thèse de doctorat. Université de Lausanne.

MoyA, A. J. 2006. On Pragmatic Functions and Their Correlation with Syntactic Functions: A Functionalist Perspective. Atlantis 28 (1): 9-28.

Neeleman, A., Titov, E., Van de Koot, H. et Vermeulen, R. 2009. A Syntactic Typology of Topic, Focus and Contrast. In J. Van Craenenbroeck (éd.), Alternatives to Cartography. Berlin: De Gruyter: 15-52.

Neeleman, A. et Vermeulen, R. 2012. The Syntactic Expression of Information Structure. In A. Neeleman et R. Vermeulen (éd.), The Syntax of Topic, Focus, and Contrast: An Interface-Based Approach. Berlin: De Gruyter: 1-38.

Prat-Sala, M., Shillcock, R. et Sorace, A. 2000. Animacy Effects on the Production of Object-Dislocated Descriptions by Catalan-Speaking Children. Journal of Child Language 27 (1) : 97-117.

Reinhart, T. 1981. Pragmatics and Linguistics. An Analysis of Sentence Topics. Philosopbica 27 (1) : 53-94.

Rickheit, G. et Habel, C. (éd.) 1995. Focus and Coberence in Discourse Processing. Berlin : De Gruyter. 
Rizzi, L. 1997. The Fine Structure of the Left Periphery. In L. HaEgeman (éd.), Elements of Grammar: Handbook in Generative Syntax. Dordrecht - Boston - Londres : Kluwer : 281-337.

Schaeffer, J. 2000. Object Scrambling and Specificity in Dutch Child Language. In S. M. Powers et C. Hamann (éd.), The Acquisition of Scrambling and Cliticization. Dordrecht - Boston - Londres: Kluwer : 71-93.

Strawson, P. F. 1964. Identifying Reference and Truth-Values. Theoria 30 (2) : 96-118.

Strömqvist, S. et Verhoeven, L. (éd.) 2004. Relating Events in Narrative. Mahwah Londres: L. Erlbaum. Vol. 2 : Typological and Contextual Perspectives.

Stutterheim, C. von et Klein, W. 1989. Referential Movement in Descriptive and Narrative Discourse. In R. Dietrich et C. F. Graumann (éd.), Language Processing in Social Context. Amsterdam - Oxford: North-Holland: 39-76.

Tolcsvai Nagy, G. 2001. A magyar nyelv szövegtana. Budapest: Nemzeti Tankönyvkiadó.

Tolcsvai Nagy, G. 2006. A szöveg. In F. Kiefer (éd.), Magyar nyelv. Budapest : Akadémiai Kiadó : $149-174$.

Tomasello, M. 1992. First Verbs: A Case Study of Early Grammatical Development. Cambridge: Cambridge University Press.

Tomasello, M. 2000. Do Young Children Have Adult Syntactic Competence? Cognition 74 (3) : 209-253.

Van Dijk, T. A. 1977. Sentence Topic and Discourse Topic. Papers in Slavic Philology 1: 49-61. En ligne à l'adresse suivante: http://www.discourses.org/OldArticles/ Sentence\%20topic\%20and\%20discourse\%20topic.pdf.

Van Dijk, T. A. 1981. Studies in the Pragmatics of Discourse. La Haye: Mouton.

Van Dijk, T. A. et Kintsch, W. 1983. Strategies of Discourse Comprehension. New YorkLondres: Academic Press.

Van Kuppevelt, J. 1995. Discourse Structure, Topicality and Questioning. Journal of Linguistics 31 (1): 109-147.

Van Rooij, R. et Schulz, K. 2017. Topic, Focus, and Exhaustive Interpretation. In C. LeE, F. Kiefer, M. Krifka (éd.), Contrastiveness in Information Structure, Alternatives and Scalar Implicatures. Cham - New York - Berlin : Springer: 63-82.

Vion, M. et ColAs, A. 1998. L'introduction des référents dans le discours en français: contraintes cognitives et développement des compétences narratives. L'année psychologique 98 (1) : 37-59.

Virtanen, T. 2004. Point of Departure: Cognitive Aspects of Sentence-Initiale Adverbials. In T. Virtanen (éd.), Approaches to Cognition through Text and Discourse. Berlin New York: De Gruyter : 79-97.

Zubizarreta, M. L. 1998. Prosody, Focus, and Word Order. Cambridge - Londres: MIT Press. 